\title{
Evaluation of a High Resolving Power Time-of-Flight Mass Spectrometer for Drug Analysis in Terms of Resolving Power and Acquisition Rate
}

\author{
Anna Pelander, ${ }^{1}$ Petra Decker, ${ }^{2}$ Carsten Baessmann, ${ }^{2}$ Ilkka Ojanperä ${ }^{1}$ \\ ${ }^{1}$ Hjelt Institute, Department of Forensic Medicine, University of Helsinki, P.O. Box 40, FI- 00014 Helsinki, Finland \\ ${ }^{2}$ Bruker Daltonik GmbH, Bremen, Germany
}

\begin{abstract}
Liquid chromatography time-of-flight mass spectrometry (LC-TOFMS) is applied increasingly to various fields of small molecule analysis. The moderate resolving power (RP) of standard TOFMS instruments poses a risk of false negative results when complex biological matrices are to be analyzed. In this study, the performance of a high resolving power TOFMS instrument (maXis by Bruker Daltonik, Bremen, Germany) was evaluated for drug analysis. By flow injection analysis of critical drug mixtures, including a total of 17 compounds with nominal masses of 212-415 Da and with mass differences of $8.8-23.5 \mathrm{mDa}$, RP varied from 34,400 to 51,900 (FWHM). The effect of acquisition rate on RP, mass accuracy, and isotopic pattern fit was studied by applying $1,2,5,10$, and $20 \mathrm{~Hz}$ acquisition rates in a 16 min gradient elution LC separation. All three variables were independent of the acquisition rate, with an average mass accuracy and isotopic pattern fit factor (mSigma) of $0.33 \mathrm{ppm}$ and 5.9, respectively. The average relative standard deviation of RP was $1.8 \%$, showing high repeatability. The performance was tested further with authentic urine extracts containing a co-eluting compound pair with a nominal mass of $296 \mathrm{Da}$ and an $11.2 \mathrm{mDa}$ mass difference. The authentic sample components were readily resolved and correctly identified by the automated data analysis. The average RP, mass accuracy, and isotopic pattern fit were $36,600,0.9 \mathrm{ppm}$, and $7.3 \mathrm{mSigma}$, respectively.
\end{abstract}

Key words: HR TOFMS, Resolving power, Acquisition rate

\section{Introduction}

T iquid chromatography time-of-flight mass spectrometry (LC-TOFMS) has become an established technique in small molecule analysis during the last decade [1]. Instrument development has been swift, and mass accuracy below $5 \mathrm{ppm}$ has become routine. Instrument robustness and stability have enabled batch analysis with fully automated data analysis [2]. In the authors' laboratory, accurate-mass based drug screening has been applied routinely to approximately 28,000 cases and various biological matrices since 2004 [2-4], proving the technique's power in terms of broad scope and efficiency.

Correspondence to: Anna Pelander; e-mail: anna.pelander@helsinki.fi
However, despite the development in mass accuracy, the resolving power (RP) of standard TOFMS instruments still remains only moderate. $\mathrm{RP}$ is defined as the capacity of a mass spectrometer to separate ions with adjacent $m / z$ [5], and is expressed as $\mathrm{m} / \Delta \mathrm{m}$ where $\mathrm{m}$ is the $\mathrm{m} / \mathrm{z}$ of an ion and $\Delta \mathrm{m}$ is the peak width measured full at $50 \%$ peak height, full width at half maximum (FWHM). Resolution (RS) is defined as the measure of separation between two peaks [5], also expressed as $\mathrm{m} / \Delta \mathrm{m}$, where $\Delta \mathrm{m}$ in this case is the difference of the two adjacent $\mathrm{m} / \mathrm{z}$. In this study, RP is applied to describe instrument performance, and RS to describe the separation requirement defined by the mass difference of specific $\mathrm{m} / \mathrm{z}$. For current standard TOFMS instruments, an RP of 10,000 is a typical performance for 
Table 1. Eight critical compound combinations in the in-house drug database analyzed by flow injection HR TOFMS

\begin{tabular}{|c|c|c|c|c|c|}
\hline Compound name & Molecular formula & {$[\mathrm{M}+\mathrm{H}]$} & Mass difference $(\mathrm{mDa})$ & $\mathrm{RS}^{\mathrm{a}}$ required & $\mathrm{RP}^{\mathrm{b}}(\mathrm{FWHM})^{\mathrm{c}}$ \\
\hline Varenicline & $\mathrm{C}_{13} \mathrm{H}_{13} \mathrm{~N}_{3}$ & 212.11822 & & & 37165 \\
\hline Mescaline & $\mathrm{C}_{11} \mathrm{H}_{17} \mathrm{NO}_{3}$ & 212.12812 & 9.9 & 21427 & 38383 \\
\hline $2-\mathrm{CT}-4^{\mathrm{d}}$ & $\mathrm{C}_{13} \mathrm{H}_{21} \mathrm{NO}_{2} \mathrm{~S}$ & 256.13658 & & & 40012 \\
\hline Clobutinol & $\mathrm{C}_{14} \mathrm{H}_{22} \mathrm{NOCl}$ & 256.14627 & 9.7 & 26434 & 34358 \\
\hline Diphenhydramine & $\mathrm{C}_{17} \mathrm{H}_{21} \mathrm{NO}$ & 256.16959 & 23.3 & 10985 & 38172 \\
\hline Melperone & $\mathrm{C}_{16} \mathrm{H}_{22} \mathrm{NOF}$ & 264.17582 & & & 44685 \\
\hline N-desmethylvenlafaxine & $\mathrm{C}_{16} \mathrm{H}_{25} \mathrm{NO}_{2}$ & 264.19581 & 20.0 & 13216 & 38165 \\
\hline Mianserin & $\mathrm{C}_{18} \mathrm{H}_{20} \mathrm{~N}_{2}$ & 265.16993 & & & 49092 \\
\hline Tetracaine & $\mathrm{C}_{15} \mathrm{H}_{24} \mathrm{~N}_{2} \mathrm{O}_{2}$ & 265.19105 & 21.1 & 12556 & 39678 \\
\hline Dibenzepin & $\mathrm{C}_{18} \mathrm{H}_{21} \mathrm{~N}_{3} \mathrm{O}$ & 296.17574 & & & 48954 \\
\hline Esmolol & $\mathrm{C}_{16} \mathrm{H}_{25} \mathrm{NO}_{4}$ & 296.18564 & 9.9 & 29918 & 44609 \\
\hline Flunitrazepam & $\mathrm{C} 16 \mathrm{H}_{12} \mathrm{~N}_{3} \mathrm{O}_{3} \mathrm{~F}$ & 314.09355 & & & 39909 \\
\hline Benorilate & $\mathrm{C}_{17} \mathrm{H}_{15} \mathrm{NO}_{5}$ & 314.10230 & 8.8 & 35897 & 41325 \\
\hline Donepezil & $\mathrm{C}_{24} \mathrm{H}_{29} \mathrm{NO}_{3}$ & 380.22202 & & & 51928 \\
\hline Oxypertine & $\mathrm{C}_{23} \mathrm{H}_{29} \mathrm{~N}_{3} \mathrm{O}_{2}$ & 380.23325 & 11.2 & 33859 & 45184 \\
\hline Flecainide & $\mathrm{C}_{17} \mathrm{H}_{20} \mathrm{~N}_{2} \mathrm{O}_{3} \mathrm{~F}_{6}$ & 415.14509 & & & 50770 \\
\hline Diltiazem & $\mathrm{C}_{22} \mathrm{H}_{26} \mathrm{~N}_{2} \mathrm{O}_{4} \mathrm{~S}$ & 415.16861 & 23.5 & 17652 & 47688 \\
\hline
\end{tabular}

${ }^{\text {a }}$ Resolution

${ }^{\mathrm{b}}$ Resolving power, measured values as average from duplicate measurement

${ }^{c}$ Full width at half maximum

d 2,5-dimethoxy-4-(i)-propylthiophenethylamine

$m / z$ 622. Despite good mass accuracy, the instruments will therefore yield large mass errors and false molecular formula assignment when co-elution of isobaric compounds with mass difference below the RP-defined threshold occurs. For example, for co-eluting $300 \mathrm{Da}$ compounds with mass differences below $30 \mathrm{mDa}$, mass errors up to $100 \mathrm{ppm}$ will occur due to sum peak formation [6].

High RP mass analyzers available for LC coupling include Fourier Transform Ion Cyclotron Resonance MS (FTICR MS) with RP above 1,000,000 [7] and orbital trapping instruments (Orbitraps) with RP up to 100,000 [8], often used as a hybrid with another mass analyzer. FTICR MS instruments are large, high cost, and unlikely to be easily adapted for routine batch analysis. Orbitraps have both high mass accuracy and high RP. However, the RP achieved is diversely proportional to acquisition rate, and the maximum RP is only obtained with long data collection times. An acquisition rate of $1 \mathrm{~Hz}$ is needed for RP 100,000 for $m / z 200$ [9]. For present-day fast chromatographic analyses with sub-2 $\mu \mathrm{m}$ particle columns, this is too slow, as typical chromatographic peak widths are $1-3 \mathrm{~s}[10,11]$. In TOFMS technology, the RP is independent of the acquisition rate. High resolving power TOFMS (HR TOFMS) would therefore be an ideal solution for identification of unknowns in complex biological samples. maXis HR TOFMS instrument was launched in 2008 by Bruker Daltonik. According to its specifications, the instrument provides an RP of 50,000 for $\mathrm{m} / \mathrm{z} 922$ due to its new technical elements, including a dual ion funnel design, an ion cooler and a dual stage reflector.

In this study, the performance of a HR TOFMS instrument was evaluated for drug analysis in an analytical toxicology context. RP was evaluated with critical compound mixtures, included in the authors' drug database, by flow injection analysis and chromatographic separation using varying acquisition rates. HR TOFMS was also compared to an ordinary TOFMS instrument with case urine samples containing an isobaric co-eluting compound pair.

\section{Experimental}

\section{Target Compounds}

The in-house target compound database used in the authors' laboratory contained 489 compounds for which a reference standard and, thus, a retention time (RT), was available. Of this group, a total of 160 belonged to two- to six-compound combinations that required an $\mathrm{RS}$ of above 10,000 by mass difference. In general, most of these mixtures could be separated chromatographically. In the category of the RS required from 10,000 to 50,000 , a total of 17 compoundsin seven two-compound mixtures and one three-compound mixture-were co-eluting, and were thus selected for the

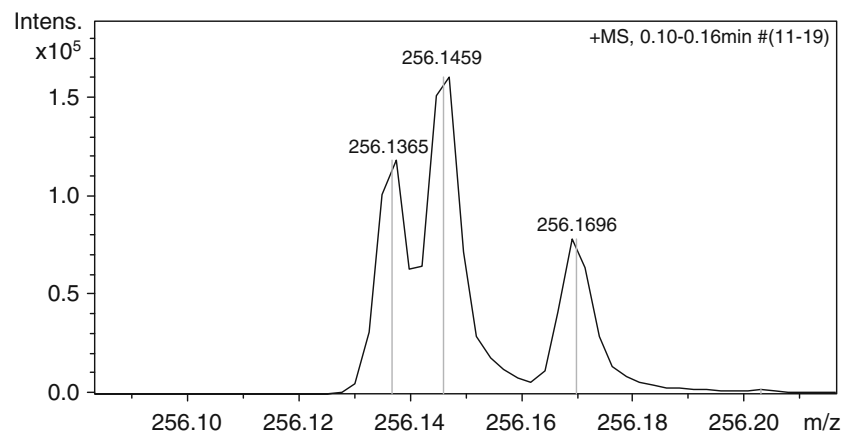

Figure 1. Mass spectrum for a three-compound mixture showing resolved peaks for 2-CT-4, clobutinol, and diphenhydramine by LC-HR TOFMS. For abbreviations and exact masses, see Table 1 
Table 2. Effect of acquisition rate on mass accuracy, isotopic pattern fit factor (mSigma) and resolving power in LC-HR TOFMS. All values are the average of triplicate measurement

\begin{tabular}{|c|c|c|c|c|c|c|c|c|c|c|c|}
\hline Compound & $\begin{array}{l}\text { Acquisition } \\
\text { rate }(\mathrm{Hz})\end{array}$ & $\begin{array}{l}\text { Mass accuracy } \\
\quad(\mathrm{ppm})\end{array}$ & mSigma & RP (FWHM) & RP RSD $\%^{a}$ & Compound & $\begin{array}{l}\text { Acquisition } \\
\text { rate }(\mathrm{Hz})\end{array}$ & $\begin{array}{l}\text { Mass accuracy } \\
(\mathrm{ppm})\end{array}$ & mSigma & $\begin{array}{c}\text { RP } \\
\text { (FWHM) }\end{array}$ & RP RSD \% \\
\hline Varenicline & 1 & 0.7 & 6.2 & 35277 & 0.8 & Mescaline & 1 & 0.9 & 8.2 & 33725 & 2.9 \\
\hline$[\mathrm{M}+\mathrm{H}]:$ & 2 & 0.5 & 3.7 & 34699 & 0.6 & {$[\mathrm{M}+\mathrm{H}]:$} & 2 & 0.2 & 7.9 & 32708 & 0.9 \\
\hline 212.11822 & 5 & 0.1 & 1.4 & 34707 & 0.4 & 212.12812 & 5 & 0.6 & 5.9 & 33851 & 4.9 \\
\hline \multirow{2}{*}{$\mathrm{RT}^{\mathrm{b}}: 3.86$} & 10 & 0.1 & 2.1 & 34266 & 1.7 & RT: 4.10 & 10 & 0.6 & 9.9 & 34031 & 2.1 \\
\hline & 20 & 0.1 & 3.3 & 34679 & 0.2 & & 20 & 0.6 & 12.2 & 35675 & 4.1 \\
\hline Melperone & 1 & 0.2 & 7.6 & 42951 & 3.8 & $\begin{array}{c}N \text {-desmethyl } \\
\text { venlafaxine }\end{array}$ & 1 & 0.4 & 4.0 & 34811 & 1.5 \\
\hline$[\mathrm{M}+\mathrm{H}]:$ & 2 & 0.2 & 4.1 & 43453 & 2.7 & {$[\mathrm{M}+\mathrm{H}]:$} & 2 & 0.4 & 4.5 & 35598 & 1.7 \\
\hline 264.17582 & 5 & 0.1 & 8.2 & 42450 & 3.6 & 264.19581 & 5 & 0.5 & 2.5 & 35335 & 2.2 \\
\hline \multirow[t]{2}{*}{ RT: 7.32} & 10 & 0.1 & 7.6 & 42804 & 3.6 & RT:7.36 & 10 & 0.5 & 4.2 & 35351 & 2.2 \\
\hline & 20 & 0.2 & 4.6 & 44389 & 0.2 & & 20 & 0.4 & 4.4 & 36264 & 2.5 \\
\hline Mianserine & 1 & 0.4 & 5.8 & 44487 & 1.6 & Tetracaine & 1 & 0.3 & 4.2 & 37063 & 1.2 \\
\hline$[\mathrm{M}+\mathrm{H}]:$ & 2 & 0.3 & 11.2 & 44250 & 5.7 & {$[\mathrm{M}+\mathrm{H}]:$} & 2 & 0.4 & 3.5 & 37020 & 1.8 \\
\hline 265.16993 & 5 & 0.5 & 9.7 & 45627 & 1.7 & 265.19105 & 5 & 0.3 & 10.8 & 36176 & 1.8 \\
\hline \multirow[t]{2}{*}{ RT:8.68 } & 10 & 0.6 & 10.3 & 47271 & 0.7 & RT: 8.78 & 10 & 0.3 & 9.6 & 36890 & 2.0 \\
\hline & 20 & 0.7 & 6.6 & 46577 & 1.9 & & 20 & 0.3 & 4.4 & 37381 & 1.1 \\
\hline Dibenzepin & 1 & 0.3 & 6.2 & 40986 & 1.6 & Esmolol & 1 & 0.4 & 8.1 & 42418 & 1.9 \\
\hline$[\mathrm{M}+\mathrm{H}]:$ & 2 & 0.3 & 7.0 & 41130 & 3.8 & {$[\mathrm{M}+\mathrm{H}]:$} & 2 & 0.2 & 5.4 & 44290 & 2.0 \\
\hline 296.17574 & 5 & 0.4 & 4.4 & 42694 & 1.6 & 296.18564 & 5 & 0.3 & 4.0 & 44567 & 3.0 \\
\hline \multirow[t]{2}{*}{ RT: 7.08} & 10 & 0.2 & 4.3 & 42839 & 3.9 & RT: 7.20 & 10 & 0.3 & 2.8 & 44614 & 3.6 \\
\hline & 20 & 0.5 & 1.6 & 43539 & 0.5 & & 20 & 0.3 & 1.8 & 45716 & 0.4 \\
\hline \multirow{5}{*}{$\begin{array}{l}\text { Flunitrazepam } \\
\text { [M + H]: } \\
314.09355 \\
\text { RT: } 12.37\end{array}$} & 1 & 0.1 & 4.8 & 45216 & 2.8 & Benorilate & 1 & 0.5 & 8.4 & 36130 & 2.6 \\
\hline & 2 & 0.4 & 7.0 & 46293 & 0.4 & {$[\mathrm{M}+\mathrm{H}]:$} & 2 & 0.5 & 6.8 & 37591 & 1.8 \\
\hline & 5 & 0.2 & 5.2 & 44209 & 1.4 & 314.10230 & 5 & 0.3 & 4.4 & 37349 & 1.8 \\
\hline & 10 & 0.04 & 4.2 & 45235 & 0.2 & RT: 12.14 & 10 & 0.2 & 4.9 & 38171 & 3.3 \\
\hline & 20 & 0.1 & 1.3 & 45744 & 1.3 & & 20 & 0.4 & 2.7 & 38096 & 0.9 \\
\hline \multirow{5}{*}{$\begin{array}{l}\text { Donepezil } \\
{[\mathrm{M}+\mathrm{H}]:} \\
380.22202 \\
\text { RT:9.16 }\end{array}$} & 1 & 0.2 & 3.0 & 46702 & 1.4 & Oxypertine & 1 & 0.5 & 6.2 & 41332 & 1.5 \\
\hline & 2 & 0.2 & 8.2 & 46853 & 2.0 & {$[\mathrm{M}+\mathrm{H}]:$} & 2 & 0.2 & 3.4 & 42657 & 0.5 \\
\hline & 5 & 0.4 & 4.5 & 47024 & 1.9 & 380.23325 & 5 & 0.2 & 1.1 & 42304 & 0.8 \\
\hline & 10 & 0.5 & 5.6 & 48486 & 1.0 & RT: 9.31 & 10 & 0.04 & 4.3 & 43553 & 1.8 \\
\hline & 20 & 0.8 & 2.2 & 48210 & 1.3 & & 20 & 0.1 & 3.3 & 43482 & 1.2 \\
\hline \multirow{5}{*}{$\begin{array}{l}\text { Flecainide } \\
{[\mathrm{M}+\mathrm{H}]:} \\
415.14509 \\
\text { RT: } 10.21\end{array}$} & 1 & 0.3 & 3.5 & 48063 & 1.6 & Diltiazem & 1 & 0.2 & 13.0 & 45385 & 1.1 \\
\hline & 2 & 0.6 & 1.7 & 49197 & 1.2 & {$[\mathrm{M}+\mathrm{H}]:$} & 2 & 0.2 & 7.7 & 45855 & 1.1 \\
\hline & 5 & 0.1 & 1.7 & 48150 & 2.6 & 415.16861 & 5 & 0.2 & 7.7 & 44429 & 1.7 \\
\hline & 10 & 0.4 & 0.9 & 49297 & 1.2 & RT: 10.12 & 10 & 0.4 & 7.6 & 46136 & 1.2 \\
\hline & 20 & 0.6 & 1.0 & 49796 & 0.5 & & 20 & 0.2 & 6.6 & 45756 & 1.2 \\
\hline \multirow{5}{*}{$\begin{array}{l}\text { 2-CT-4 } \\
{[\mathrm{M}+\mathrm{H}]:} \\
256.13658 \\
\text { RT: } 9.24\end{array}$} & 1 & 0.03 & 9.0 & 34973 & 2.4 & Clobutinol & 1 & 0.2 & 7.4 & 39972 & 0.8 \\
\hline & 2 & 0.4 & 6.9 & 34610 & 0.5 & {$[\mathrm{M}+\mathrm{H}]:$} & 2 & 0.4 & 7.7 & 41212 & 0.7 \\
\hline & 5 & 0.3 & 8.1 & 34648 & 3.5 & 256.14627 & 5 & 0.2 & 6.2 & 40588 & 2.8 \\
\hline & 10 & 0.3 & 5.2 & 35667 & 1.4 & RT: 8.44 & 10 & 0.2 & 6.1 & 41100 & 0.7 \\
\hline & 20 & 0.3 & 3.5 & 36350 & 2.3 & & 20 & 0.3 & 2.8 & 41509 & 1.0 \\
\hline \multirow{5}{*}{$\begin{array}{l}\text { Diphenhydramine } \\
{[\mathrm{M}+\mathrm{H}]:} \\
256.16959 \\
\text { RT: } 8.76\end{array}$} & 1 & 0.3 & 3.7 & 36421 & 3.9 & & & & & & \\
\hline & 2 & 0.2 & 12.1 & 35479 & 3.8 & & & & & & \\
\hline & 5 & 0.1 & 17.8 & 36003 & 3.2 & & & & & & \\
\hline & 10 & 0.4 & 11.9 & 34807 & 0.7 & & & & & & \\
\hline & 20 & 0.5 & 17.6 & 34658 & 0.9 & & & & & & \\
\hline
\end{tabular}

a Relative standard deviation

${ }^{\mathrm{b}}$ Retention time in $\mathrm{min}$

For other abbreviations, see Table 1

study. The compounds with their RT and the required RS are shown in Table 1. In addition, six compounds in three twocompound combinations required an RS above 100,000. However, the latter compounds could be chromatographically separated. RS from 50,000 to 100,000 was required for
18 compounds in nine two-compound combinations. Eight pairs could be readily separated by chromatography, and only one pair, prazosin and quetiapine, eluted close to each other (RT 12.13 and $12.44 \mathrm{~min}$, respectively), requiring an RS of 51,915 .

Table 3. Mass accuracy, resolving power, and isotopic pattern fit factor (mSigma) for dibenzepin and a doxepin metabolite in 5 authentic autopsy urine extracts by LC-HR TOFMS. For abbreviations, see Table 1

\begin{tabular}{|c|c|c|c|c|c|c|}
\hline Case & $\begin{array}{l}\text { Mass accuracy doxepin } \\
\text { metabolite ppm }\end{array}$ & $\begin{array}{l}\text { Mass accuracy } \\
\text { dibenzepin ppm }\end{array}$ & $\begin{array}{l}\text { RP (FWHM) doxepin } \\
\text { metabolite }\end{array}$ & $\begin{array}{c}\text { RP (FWHM) } \\
\text { dibenzepin }\end{array}$ & $\begin{array}{l}\text { mSigma doxepin } \\
\text { metabolite }\end{array}$ & $\begin{array}{l}\text { mSigma } \\
\text { dibenzepin }\end{array}$ \\
\hline 6072 & 0.7 & 0.1 & 42579 & 34631 & 2.5 & 1.8 \\
\hline 6101 & 0.3 & 2.4 & 45716 & 28520 & 4.4 & 7.0 \\
\hline 6102 & 1.7 & 0.4 & 33893 & 37262 & 15.1 & 2.9 \\
\hline 6183 & 0.7 & 1.2 & 39739 & 35875 & 10.5 & 4.2 \\
\hline 6197 & 0.5 & 1.0 & 36330 & 31833 & 12.0 & 12.8 \\
\hline
\end{tabular}


(a)

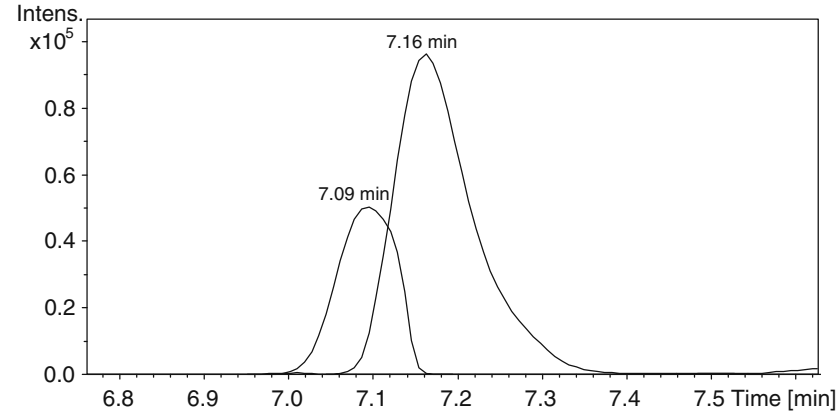

(b)

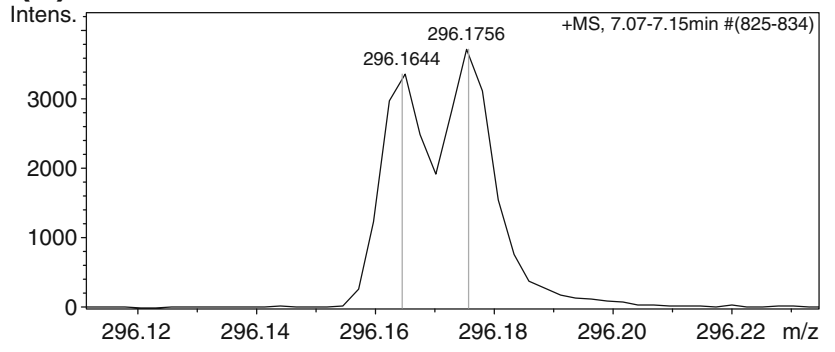

Figure 2. (a) Extracted ion chromatograms for dibenzepin (RT $7.09 \mathrm{~min}$ ) and doxepin metabolite (RT $7.16 \mathrm{~min}$ ) by LCHR TOFMS, using a 3 mDa mass window. (b) Mass spectrum for $\mathrm{m} / \mathrm{z} 296$ from the co-eluting part of the two EIC peaks in (a). The exact masses for doxepin metabolite and dibenzepin were 296.1645 and 296.1757 [M + H], respectively

\section{Liquid Chromatography Time-of-Flight Mass Spectrometry}

The liquid chromatograph was a Dionex (Sunnyvale, CA, USA) Ultimate 3000 series RSLC instrument including a vacuum degasser (SRD-3600), autosampler (WPS 3000TRS), binary pump (HPG 3400RS), and column oven (TTC 3000 RS). Separation was performed in gradient mode with a Phenomenex (Torrance, CA, USA) Luna PFP(2) $100 \times 2 \mathrm{~mm}(3 \mu \mathrm{m})$ column and a $4 \times 2 \mathrm{~mm}$ pre-column at $40{ }^{\circ} \mathrm{C}$. Mobile phase components were $2 \mathrm{mM}$ ammonium acetate in $0.1 \%$ formic acid (A) and methanol (B). The flow rate was $0.3 \mathrm{~mL} / \mathrm{min}$. The proportion of methanol was increased from $10 \%$ to $40 \%$ in $5 \mathrm{~min}$, to $75 \%$ in $13.5 \mathrm{~min}$, to $95 \%$ in $14 \mathrm{~min}$, and held at $95 \%$ for $2 \mathrm{~min}$. The post-time was $4 \mathrm{~min}$ and injection volume $1 \mu \mathrm{L}$.

The mass analyzer was a Bruker Daltonik (Bremen, Germany) maXis TOFMS instrument with an electrospray ionization (ESI) source and a six-port divert valve. The instrument controls were performed via Compass ver. 1.3 (Bruker Daltonik) software package. The specified RP of the instrument was 50,000 by FWHM for $m / z 922$. The instrument was operated in positive ion mode with an $\mathrm{m} / \mathrm{z}$ range of 50450. The nebulizer gas pressure was 2.8 bar, and the drying gas flow was $9 \mathrm{~L} / \mathrm{min}$. The drying temperature was $200{ }^{\circ} \mathrm{C}$. The acquisition rate varied from $1 \mathrm{~Hz}$ to $20 \mathrm{~Hz}$, corresponding to spectra summations of 12,346 and 617 .
External instrument mass-scale calibration was performed in high precision calibration (HPC) mode both before sequence start and post-run for the individual data set in automated way with mixed sodium formate/acetate clusters using a solution of $10 \mathrm{mM}$ sodium hydroxide in isopropanol/ water $(1: 1, \mathrm{vol} / \mathrm{vol})$ containing $0.15 \%$ acetic acid and $0.05 \%$ formic acid. The calibrant solution was continuously pumped with a flow of $10 \mu \mathrm{L} /$ min through a $20 \mu \mathrm{L}$ loop mounted on a six-port valve built in the MS system. A timesegment based MS acquisition method with automatic valve switching was used to introduce the calibrant solution into the LC flow path during the chromatographic dead-time. Additional post-run internal calibration of the individual data sets was performed using methyl stearate. Portions of a methyl stearate solution (1 $\mathrm{mg} / \mathrm{mL}$ in isopropanol) were applied to a calibrant reservoir fixed within the ESI spray chamber to give a constantly present calibrant signal.

For the flow injection analysis the separation column was removed from the LC system and the compound mixtures were injected into a flow of $0.3 \mathrm{~mL} / \mathrm{min}$ of $50 \%$ eluent $\mathrm{B}$ with acquisition rate of $2 \mathrm{~Hz}$. The flow injection analysis of the critical combinations was performed in duplicate. The test solutions were prepared in methanol to include the critical combinations to be resolved in a concentration of 1$10 \mu \mathrm{g} / \mathrm{mL}$, depending on the analyte. The concentrations were selected to produce similar ion abundances.

The effect of acquisition rate on RP, mass accuracy and isotopic pattern fit was studied via chromatographic separation

(a)

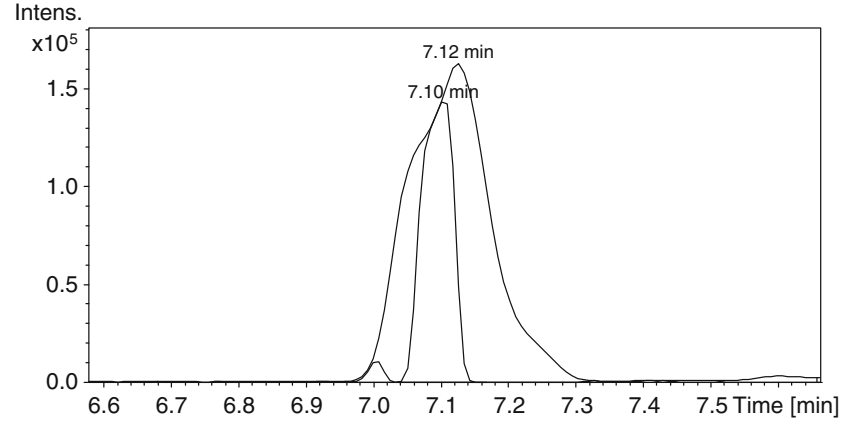

(b)

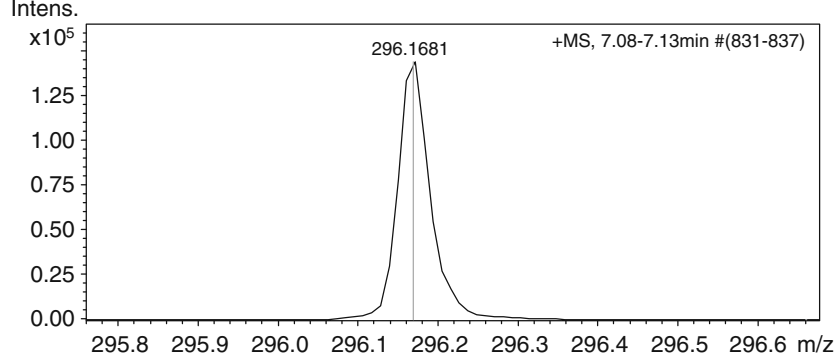

Figure 3. (a) Extracted ion chromatograms for dibenzepin (RT $7.10 \mathrm{~min}$ ) and doxepin metabolite (RT $7.12 \mathrm{~min}$ ) by LCTOFMS, using a $3 \mathrm{mDa}$ mass window. (b) Mass spectrum for $\mathrm{m} / \mathrm{z} 296$ from the co-eluting part of the two EIC peaks in (a). A single sum peak is observed in the mass spectrum 
of a methanolic solution containing all 17 compounds with a concentration of $1 \mu \mathrm{g} / \mathrm{mL}$. The acquisition rates applied were 1 , 2, 5, 10, and $20 \mathrm{~Hz}$. All experiments were performed in triplicate.

\section{Authentic Case}

In the authors' laboratory, dibenzepin is used as the internal standard (ISTD) in screening applications. The molecular formula and exact mass of dibenzepin are $\mathrm{C}_{18} \mathrm{H}_{21} \mathrm{~N}_{3} \mathrm{O}$ and $296.1757[\mathrm{M}+\mathrm{H}]$, respectively. A doxepin metabolite with molecular formula $\mathrm{C}_{19} \mathrm{H}_{21} \mathrm{NO}_{2}$ and exact mass of $296.1645[\mathrm{M}+\mathrm{H}]$ is known to co-elute with dibenzepin with the standard LC-method used. The mass difference is $11.2 \mathrm{mDa}$, which would require RS of 26,400 for differentiation. Five autopsy urines known to contain doxepin and metabolites were selected to test the performance of HR TOFMS at an acquisition rate of $2 \mathrm{~Hz}$ with the authentic biological samples. The sample preparation was based on mixed-mode solid-phase extraction, as described in detail elsewhere [2]. The conventional TOFMS instrument used for performance comparison in the authentic sample analysis was a micrOTOF (Bruker Daltonik), which is used in the laboratory's routine applications as described earlier [2].

\section{Data Analysis}

Data analysis was mainly performed with Target Analysis ver. 1.2 software (Bruker Daltonik) using automated reverse search of a database containing the RT, exact mass $[\mathrm{M}+\mathrm{H}]$, and molecular formula of the target compounds. All mass accuracy and isotopic pattern fit values listed in the tables were collected from the results reports generated by this software. The RP values were picked manually from the processed data files.

\section{Results and Discussion}

Flow injection analysis was performed in order to test the RP of the instrument without chromatographic separation. Table 1 shows the critical drug combinations identified in the authors' drug database, the required RS by mass difference, and the measured RP (FWHM) for each ion. The required RS varied from approximately 11,000 (clobutinol-diphenhydramine) to 36,000 (flunitrazepam-benorilate), while the measured RP varied from 34,400 to 51,900. All combinations were resolved with success. As the instrument specification was 50,000 for $\mathrm{m} / \mathrm{z} 922$, the measured 51,900 for $380 \mathrm{Da}$ (donepezil) shows an excellent level of performance, as in TOFMS technology the peak width of the TOF is approximately proportional to the mass, where as the detector peak width is constant. Therefore, with increasing $\mathrm{m} / \mathrm{z}$ the detector peak width contributes less to resulting peak width and the resolving power increases with higher $\mathrm{m} / \mathrm{z}$. Figure 1 shows the result for a three-compound mixture with RP from 34,400 to 40,000 . The first two compounds, 2-CT-4 and clobutinol, had a mass difference of only $9.7 \mathrm{mDa}$. In automated data analysis, both compounds were readily identified, although the ions did not show full baseline separation. The separation of the two ions was adequate for identification purposes, but close to the performance limits of the instrument. The flow injection analysis experiments proved the instrument specifications to conform to its actual performance.

Table 2 shows the effect of the acquisition rate on RP, mass accuracy and isotopic pattern fit expressed as mSigma. The acquisition rate varied from $1 \mathrm{~Hz}$ to $20 \mathrm{~Hz}$. The RP was stable and independent of the acquisition rate: the average relative standard deviation (RSD\%) of the triplicate analyses was $1.8 \%$, varying from $0.2 \%$ (flunitrazepam, $10 \mathrm{~Hz}$ ) to $5.7 \%$ (mianserin, $2 \mathrm{~Hz}$ ). The mass accuracy was always below $1 \mathrm{ppm}$ with an average of $0.33 \mathrm{ppm}$. The acquisition rate had no effect on mass accuracy. The SigmaFit value is based on the relative mean square of the difference of an experimental mass spectrum from the theoretical isotopic pattern of a specific molecular formula. The instrument specification does not include mSigma threshold for molecular formula assignment. Kind and Fiehn have proposed a $5 \%$ upper limit for isotopic abundance pattern error in molecular formula assignment [12]. SigmaFit is not expressed in \%, but a Sigma value of $50 \mathrm{mSigma}$ (0.05 Sigma) approximates closely to the 5\% definition. Therefore, the lower the mSigma value, the more precise the fit. In our earlier study of the effect of accurate mass and isotopic pattern on the accuracy of identification, the average mSigma value in authentic urine samples for true positives was 6.6, and a threshold of 30 has been applied on a routine basis [2]. In the current study, the isotopic pattern fit was independent of the acquisition rate, with an average value of 5.9 .

Table 3 shows mass accuracy, RP, and mSigma for dibenzepin and a doxepin metabolite, forming a co-eluting pair with a mass difference of $11.2 \mathrm{mDa}$, in five authentic urine samples following LC separation. The measured RP for the adjacent ions varied from 28,500 to 45,700 , while the

Table 4. The distribution of database compounds according to RS requirement. Sum exceeds the total of 160 (see Experimental section), as some compounds appeared in more than one category. For abbreviations, see Tables 1 and 2

Number of compounds with RT in in-house database

\begin{tabular}{ccccc}
\hline All & Requiring RS $10,000-50,000$ & Requiring RS $50,000-100,000$ & Requiring RS $>100,000$ & Chromatographic separation \\
\hline 489 & 125 & 16 & 6 & Yes \\
& 17 & 2 & 0 & No \\
\hline
\end{tabular}


required RS value was 26,400 . The average was 36,000 . Both dibenzepin and the doxepin metabolite were correctly identified in automated data analysis. Figure 2 shows the extracted ion chromatograms for the two compounds in a $3 \mathrm{mDa}$ mass window and the mass spectrum with the separation of the ions. In addition, the extract was analyzed with an ordinary TOFMS instrument with moderate RP (Figure 3). A single sum peak is seen in the mass spectrum, resulting in mass errors of -7.3 and $+3.6 \mathrm{mDa}$ for dibenzepin and the doxepin metabolite, respectively. The difference in performance between the two instruments shown in Figures 2 and 3 clearly proves the power and importance of high resolving power: in Figure 3, the shapes of the chromatograms hint at serious overlapping, but the mass spectrum appears perfectly normal when visually inspected. However, both compounds would have given a false negative result when operating in a $3 \mathrm{mDa}$ window. The average mass accuracy for the two compounds in five cases was $0.9 \mathrm{ppm}$, which is somewhat higher than the reference standard average (Tables 2 and 3), but still below $1 \mathrm{ppm}$. However, a slight increase in mass accuracy values is normal when switching from the analysis of pure substances to biological matrix. The mSigma average was 7.3.

The question of correct molecular formula assignment has been discussed in the literature in terms of mass accuracy, resolving power, and isotopic pattern fit $[9,12-$ 14]. It has been shown that correct formula assignment requires all of these parameters with very strict criteria.

Both Kellmann et al. [9] and Nielen et al. [13] have shown that accurate mass alone is insufficient for molecular formula assignment in pesticide and hormone trace analysis, and that high resolving power is required. They both compared the performance of TOFMS with orbital trapping instruments that provide RP up to 100,000 . Kellmann et al. recommended adjusting the RP according to matrix type: for less complex matrices they suggested an RP of 18,00025,000 ; for highly complex extracts an RP of 35,000 50,000 , or even up to $70,000-100,000$. They used a straightforward and simple, yet non-selective sample preparation method. The RP achieved in our study is well in line with the 50,000 threshold. The remaining gap to RP 100,000 can be partially compensated by selective sample preparation and increased chromatographic resolution.

In our database of 489 compounds, 142 belonged to the group requiring a resolution of 10,000-50,000, which represents nearly one-third of the database. However, only 17 of these compounds, representing approximately $3.5 \%$ of the database, eluted in close proximity to each other. Only two compounds, prazosin and quetiapine, forming $0.4 \%$ of the database, would have been beyond the performance of HR TOFMS, as they eluted close to each other and had a mass difference of $7.4 \mathrm{mDa}$, requiring an RS above 50,000. This demonstrates that in targeted screenings, large errors in mass due to sum peak formation are a real risk but usually avoidable by employing adequate chromatography and selective sample preparation. In addition, there are combinations for which even an RP of 100,000 is by itself insufficient: for example, melperone $\left(\mathrm{C}_{16} \mathrm{H}_{22} \mathrm{NOF}, \mathrm{m} / z 264.17582[\mathrm{M}+\mathrm{H}]\right)$ and nortriptyline $\left(\mathrm{C}_{19} \mathrm{H}_{21} \mathrm{~N}, \mathrm{~m} / \mathrm{z} 264.17470[\mathrm{M}+\mathrm{H}]\right)$ differ only by $1.1 \mathrm{mDa}$ in mass and, thus, would require RS 231,700 for differentiation by mass. Fortunately, they are easily separated by chromatography, but this example demonstrates the potential for false negatives when the matrix components are unknown. The distribution of RS requirements within the in-house database is presented in Table 4. For unambiguous compound detection and identification, complementary and orthogonal information should be considered rather than just focusing on the increase of the RP of the analytical technique. For example, in the extreme case of co-eluting compounds of the same sum formula, even an unlimited RP will not help in discrimination between the compounds, but additional information like fragment/qualifier ions is needed.

Kind and Fiehn have demonstrated the importance of accurate isotopic pattern fit for correct molecular formula assignment [12]. They even state that mass accuracy was found to be a less important parameter than correct isotopic ratios for molecular formula assignment [14]. HR TOFMS instrument performance was stable and independent of the acquisition rate in this respect as well, as the acquisition rate had no effect on the mSigma. For Orbitraps, it has been recently indicated that spectral accuracy is less stable with increasing RP [15]. Therefore, when the three critical aspects- RP, isotopic pattern fit, and mass accuracy—are evaluated as a whole, HR TOFMS has clear benefits due to its stability and acquisitionrate-independent performance. The most challenging combinations still remain, however, beyond the performance of any instrument other than the FTICR MS.

\section{Conclusions}

HR TOFMS instrument performance was stable, repeatable, and independent of the acquisition rate, making it amenable to fast chromatography. The instrument specification of RP 50,000 was found to be realistic in drug analysis. Analysis of case urine samples showed the necessity of high RP in solving real-life separation problems. Further studies will be needed to determine the tandem MS properties of the instrument and its applicability to structural studies.

\section{References}

1. Ferrer, I., Thurman, E.M.: Liquid Chromatography Time-of-Flight Mass Spectrometry. Wiley, Hoboken (2009)

2. Ojanperä, S., Pelander, A., Pelzing, M., Krebs, I., Vuori, E., Ojanperä, I.: Isotopic pattern and accurate mass determination in urine drug screening by liquid chromatography/time-of-flight mass spectrometry. Rapid Commun. Mass Spectrom. 20, 1161-1167 (2006)

3. Pelander, A., Ristimaa, J., Rasanen, I., Vuori, E., Ojanperä, I.: Screening for basic drugs in hair of drug addicts by liquid chromatography/time-of-flight mass spectrometry. Ther. Drug Monit. 30, 717-724 (2008)

4. Pelander, A., Ristimaa, J., Ojanperä, I.: Vitreous humor as an alternative matrix for comprehensive drug screening in post-mortem toxicology by liquid chromatography time-of-flight mass spectrometry. J. Anal. Toxicol. 34, 312-318 (2010) 
5. Sparkman, O.D.: Mass Spectrometry Desk Reference, 2nd edn, p. 55. Global View Publishing, Pittsburgh (2006)

6. Sleno, L., Volmer, D.A., Marshall, A.G.: Assigning product ions from complex MS/MS spectra: the importance of mass uncertainty and resolving power. J. Am. Soc. Mass Spectrom. 16, 183-198 (2005)

7. Cottingham, K.: Product review: FTMS: overcoming challenges. Anal. Chem. 78, 655-657 (2006)

8. Makarov, A., Denisov, E., Kholomeev, A., Balschun, W., Lange, O., Strupat, K., Horning, S.: Performance evaluation of a hybrid linear ion trap/orbitrap mass spectrometer. Anal. Chem. 78, 2113-2120 (2006)

9. Kellmann, M., Muenster, H., Zomer, P., Mol, H.: Full scan MS in comprehensive qualitative and quantitative residue analysis in food and feed matrices: how much resolving power is required? J. Am. Soc. Mass Spectrom. 20, 1464-1476 (2009)

10. Rainville, P.D., Stumpf, C.L., Shockcor, J.P., Plumb, R.S., Nicholson, J.K.: Novel application of reversed-phase UPLC-oaTOF-MS for lipid analysis in complex biological mixtures: a new tool for lipidomics. $J$. Proteome Res. 6, 552-558 (2007)
11. Wu, T., Wang, C., Wang, X., Xiao, H., Ma, Q., Zhang, Q.: Comparison of UPLC and HPLC for Analysis of 12 Phthalates. Chromatographia 68, 803-806 (2008)

12. Kind, T., Fiehn, O.: Metabolomic database annotations via query of elemental compositions: mass accuracy is insufficient even at less than 1 ppm. BMC Bioinform. 7, 234 (2006)

13. Nielen, M.W., van Engelen, M.C., Zuiderent, R., Ramaker, R.: Screening and confirmation criteria for hormone residue analysis using liquid chromatography accurate mass time-of-flight, fourier transform ion cyclotron resonance and orbitrap mass spectrometry techniques. Anal. Chim. Acta 586, 122-129 (2007)

14. Kind, T., Fiehn, O.: Seven golden rules for heuristic filtering of molecular formulas obtained by accurate mass spectrometry. BMC Bioinform. 8, 105 (2007)

15. Erve, J.C., Gu, M., Wang, Y., DeMaio, W., Talaat, R.E.: Spectral accuracy of molecular ions in an LTQ/orbitrap mass spectrometer and implications for elemental composition determination. J. Am. Soc. Mass Spectrom. 20, 2058-2069 (2009) 